\title{
The assessment and management of dementia
}

\section{Z Vally MA(ClinPsy)}

To cite this article: Z Vally MA(ClinPsy) (2010) The assessment and management of dementia, South African Family Practice, 52:5, 392-395, DOI: 10.1080/20786204.2010.10874014

To link to this article: http://dx.doi.org/10.1080/20786204.2010.10874014

() 2010 SAAFP. Published by Medpharm.

Published online: 15 Aug 2014.

Submit your article to this journal $\sqsubset$

凹 Article views: 47

Q View related articles $\sqsubset$ 


\title{
The assessment and management of dementia
}

\begin{abstract}
South African family practitioners can expect to contend with rising rates of dementia as the aged segment of the population grows larger and HIV-related neuropsychiatric complications manifest with increasing prevalence. Family practitioners are often the first professional consulted by families concerned with a family member's suspected dementia. This fortunate position facilitates the early identification of cognitive difficulties, which, in turn, greatly increases the likelihood of optimal management. Dementia is best managed with a multidisciplinary-team approach that sees family practitioners fulfilling the role of key support to families and of the coordinators of multidisciplinary referrals and inputs. This article reviews the key diagnostic features of dementia and provides suggestions that may be utilised by physicians in the basic assessment of these features. It concludes with a summary of the most appropriate management plan to be adopted by family practitioners.
\end{abstract}

(P) Peer reviewed. (Submitted: 2010-01-23, Accepted: 2010-02-20). ๑ SAAFP

SA Fam Pract 2010;52(5):392-395

\section{Introduction}

Whilst some individuals progress through life and maintain relatively high levels of cognitive function, for most, some form of cognitive decline in later life is expected. Such changes vary among aging individuals but they may include some slowing of reaction time, a decrease in the capacity for memory and a reduction in visuospatial ability. Importantly, however, for this majority of older individuals who experience some alteration in neurocognitive functioning, the change is mild and not sufficiently powerful to dissolve their capacity for normal social and occupational functioning. In contrast, the dramatic decline that results from dementia sees a global loss of normal cognitive function. This article outlines suggestions for the effective assessment and management of dementia for general family practitioners.

\section{Dementia in South Africa}

The aging segment of the population is expanding worldwide; South Africa, in particular, has one of the most rapidly aging populations in Africa. ${ }^{2} \mathrm{~A}$ substantial increase in the proportion of aging individuals who are likely to develop dementia can consequently be expected over the next 40 years. More than 30 million people worldwide are currently living with dementia. This figure, however, is expected to rise to 100 million by the year 2050, when more than 1 in 5 individuals will be over the age of $60 .{ }^{3}$ This worldwide phenomenon sees an exponential growth in the over-65 age category, whilst lowered fertility rates and high rates of infant mortality will most likely render South Africa especially affected.

Additionally, cognitive impairment and later dementia are a common associated symptom of the AIDS stage of HIV disease. Many studies have found moderate to severe cognitive deficits in the majority of HIV-positive individuals sampled. ${ }^{4}$ Sub-Saharan Africa remains the region most heavily affected by HIV worldwide, accounting for over twothirds $(67 \%)$ of all people living with HIV and for nearly threequarters $(72 \%)$ of AIDS-related deaths in $2008 .{ }^{5}$ Given this increasingly high prevalence of HIV illness, it is expected that South African physicians will most likely encounter similarly rising rates of dementing disease and the full spectrum of HIV-related neuropsychiatric symptoms.

\section{Diagnosis of dementia}

\section{Early identification}

Family practitioners are often the first port of call for families seeking advice regarding a family member's suspected dementia. Although the full diagnostic criteria for dementia may not yet be warranted at this early stage, the diagnostic label of 'mild cognitive impairment' (MCl) may be used for those individuals who evidence objective and subjective memory difficulties (preferably corroborated by a collateral source) in the presence of normal general cognitive functioning and the intact activities of daily living but who 
are not yet demented. It appears, however, that patients with $\mathrm{MCl}$ are at increased risk of the later development of Alzheimer's disease (AD). ${ }^{6}$ The early detection of $\mathrm{MCl}$ is therefore imperative in delaying this seemingly natural progression towards the development of $A D$ and it is family practitioners who may be in the best position to facilitate this early response.

\section{Diagnostic criteria}

The essential feature of a dementia is the development of multiple cognitive deficits that include memory impairment and at least one of the following cognitive disturbances: aphasia, apraxia, agnosia or a disturbance in executive functioning. The cognitive deficits should be sufficiently severe to cause impairment in occupational or social functioning and should represent a decline from a previously higher level of functioning. ${ }^{7}$ Once the presence of dementia is established, an attempt should be made to identify its aetiology through its history, a thorough clinical examination, neuropsychological assessment and, where feasible, imaging and laboratory studies. None of the currently available biological markers, however, is useful for establishing the diagnosis of any of the most common forms of dementia with certainty: $A D$, vascular dementia, dementia with Lewy bodies and frontotemporal dementia. Clinicians should therefore rely on clinical criteria to make a diagnosis.

\section{Assessment of dementia}

Several instruments that facilitate the initial screening of patients suspected of being at risk of dementia exist. The most common instrument employed for this purpose is the mini-mental status examination. Despite widespread use in South Africa, however, its psychometric validity for the local population is unknown. If this initial assessment evidences cognitive fall-off, it is therefore recommended that further neuropsychological assessment of memory, language, visuospatial and executive functioning be facilitated via referral to a neurologist or neuropsychologist.

Lentegeur Hospital utilises a 'brief' assessment for this purpose. The assessment is designed to assess the following cognitive domains to identify patients suffering from dementia: attention or concentration, orientation, speech and language, executive planning, visuospatial skills, psychomotor functioning and problem-solving ability. Tests are selected for inclusion in the battery on the basis of their capacity to capture the specific diagnostic deficits in the nosological criteria for dementia. Table 1 lists the neuropsychological assessment techniques that may be administered during the clinical interview to aid the diagnosis of dementia. These include the identification of whether patients have lost the ability for adaptive functioning. In other words, are they still able to carry out tasks encountered in daily living, such as bathing, toileting and grooming? It is important, however, that the assessed

Table 1: Diagnostic features and interview assessment techniques

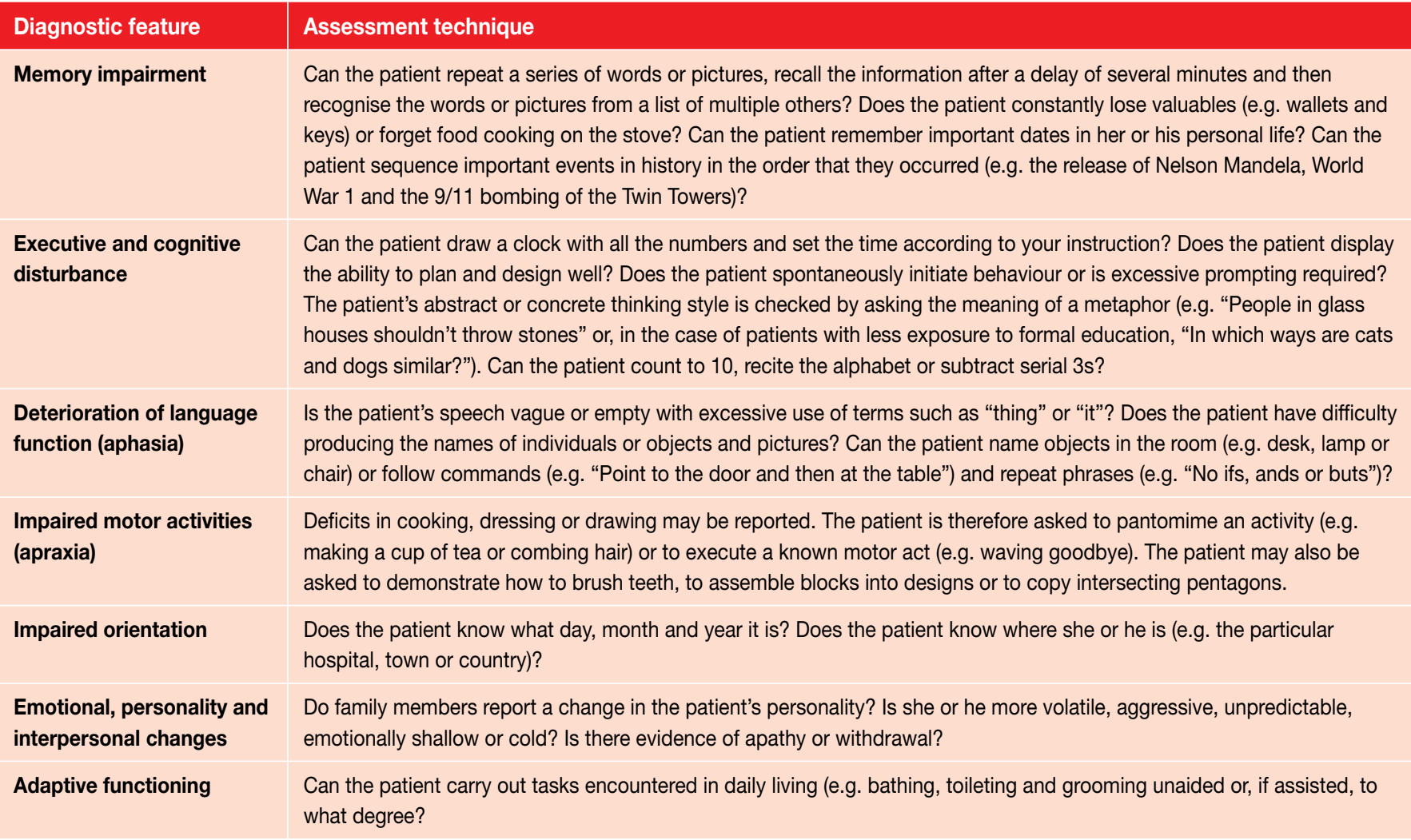


behaviours should be those expected of individuals at their particular age given their specific cultural setting. Such information is usually obtained by perusing the patients' history or interviewing a caregiver.

\section{Special investigations and referrals}

All patients with suspected dementia should undergo thorough physical and neurological examinations. Medical illnesses that can result in dementia include thyroid disease, atherosclerotic-vascular disease, collagen-vascular disease (such as systemic lupus erythematosus) and infections. Clinicians should be attentive to the skin for the thinning of hair and eyebrows, spider hemangiomata, palmar erythema, malar rash or Kaposi's sarcoma. Heart sounds, liver texture and size, and the presence of fever, hypertension or lymphadenopathy may also provide important diagnostic clues. Visual-field defects, eye-movement abnormalities, facial asymmetry, dysarthria, focal weakness and spasticity may indicate the presence of focal-brain or brain-stem lesions due to stroke, tumour or infectious disease, such as toxoplasmosis. Where feasible, patients with clinicaldementia syndrome should undergo structural-brain imaging with non-contrast computed tomography or magnetic resonance imaging to evaluate for focal lesions, deep white-matter ischaemic changes and regions of atrophy. Certain laboratory tests are valuable for the initial screening of patients with cognitive changes. In particular, thyroidfunction tests (testing for the thyroid-stimulating hormone and free T4) and the checking of vitamin B levels should be checked in patients with cognitive complaints. In cases of borderline $B$ deficiency, elevated levels of homocysteine and methylmalonic acid enhance the sensitivity of $B$ levels. Patients with risk factors for HIV or venereal syphilis should undergo appropriate tests. ${ }^{2}$

\section{Management of dementia}

It is preferable that the management of dementia be multidisciplinary in nature. Family practitioners should contain and alleviate the family's grief and anxiety by providing education on the patient's diagnosis and an attentive and supportive space within which to voice concerns and fears. They will furthermore most likely be responsible for engaging the family in conversations around care-giving arrangements or possible institutionalisation and for coordinating referrals to other disciplines. Family practitioners should also bear the following in mind:

- It is preferable to delay institutionalisation as long as this is feasible and within the caregiver's capacity to do so, since patients with dementing diseases enjoy a longer life expectancy when not institutionalised.

- Encourage the performance of activities of daily living and the provision of opportunities for cognitive exercise

\section{Table 2: Classification of dementia drugs ${ }^{8}$}

\author{
A. Cholinergic deficiency \\ 1. Pre-synaptic agents: \\ - These drugs either increase the synthesis of acetylcholine \\ (Ach) or promote the release of Ach: \\ - Ach precursors: Choline or lecithin \\ - Increased Ach synthesis/release: Piracetam, \\ 4-amino-pyridine \\ - Selective muscarinic 2 receptor antagonists: Gallamine, \\ scopolamine \\ - Anti-choline esterases: Tetrahydroaminoacridine
}

\section{Synaptic agents:}

These drugs limit the removal of Ach from the synapse - choline esterase inhibitors (CHEIs), e.g. physostigmine and metrifonate:

- First generation: Tacrine

- Second generation:

- Donepezil

- Galantamine

- Rivastigmine

- Butyrylcholinesterases

- Nerve growth factor gene delivery

3. Post-synaptic agents:

Cholinergic agents acting directly on the muscarinic receptors: Pilocarpine

Oxotremorine

\section{B. Antioxidants}

1. Selegeline

2. Alpha-tocopherol

\section{Anti-amyloids}

1. Statins

2. $A B$ vaccination

3. Immunotherapy - bapineuzumab and A B peptide antibody

4. Secretase effectors:

i) LY450139, a non-selective $\gamma$ secretase inhibitor

ii) Memapsin 2 ( $B$ secretase)

5. Selective amyloid-lowering agents:

i) Tarenflurbil $-\alpha \gamma$ secretase modulator

ii) Non-steroidal anti-inflammatory drugs - regulate $₫$ secretase

\section{Exocitoxicity}

Glutamate toxicity to neurons:

i) Memantine - N-methyl d-aspartate receptor antagonist

in the patient, such as reading and writing, listening to the radio, watching television, listening to music, participating in conversations with others and perhaps doing puzzles and playing board-games if this is possible.

- Refer the family to a psychologist or specialist support group, such as Dementia SA, for psychotherapeutic intervention or counselling. Such intervention is beneficial in improving the caregiver's quality of life and greatly builds the caregiver's psycho-emotional resources, increasing the likelihood of managing the challenges associated with caring for a patient with dementia. 
- Refer the patient to a neurologist to assist in the identification of signs of Parkinson's disease, strokes, tumours and other medical conditions that may impair memory and thinking (such as head injuries) and of physical function.

- Refer the patient to a psychiatrist to exclude other underlying or co-morbid psychiatric disorders, such as depression, to determine which thinking and memory functions may be affected and to what degree and to provide ongoing management in the event that psychiatric symptoms have begun to emerge.

The development of the pharmacological treatment of dementia is problematic, as clinical improvement is not well understood or defined. Table 2 outlines a classification of all the drugs that may potentially have a clinical effect on patients suffering from dementia.

Of all these drugs, only the following four are registered for clinical use in dementia in South Africa:

- Donepezil (Aricept ${ }^{\circledR}$ )

- Rivastigmine (Excelon $\left.{ }^{\circledR}\right)$

- Galantamine $\left(\right.$ Reminyl $\left.{ }^{\circledR}\right)$

- Memantine $\left(\right.$ Ebixa $\left.^{\circledR}\right)$

Patients with a diagnosis of $A D$ may also require antidepressants (sertraline, escitalopram, trazodone), atypical antipsychotics (risperidone, olanzapine, quetiapine) or anticonvulsants (carbamazepine, valproic acid).

The following practical approach is advocated in the management of dementia: ${ }^{8}$

- A thorough clinical examination and special investigations to ascertain the type of dementia.

- The treatment of underlying conditions and other chronic diseases, such as hypertension and diabetes.

- The severity scoring of the dementia.

- The prescription, initially, of either a second-generation or an exocitotoxicity drug (memantine).

- The prescription, initially, of a low dose and the evaluation of side effects and tolerability in the first month and of efficacy over the next two months.

- If not efficacious, an increase in the dose to the maximum recommended dose.

- The addition of either a CHEl or memantine to the regimen, depending on which one was started first.

\section{Conclusion}

Dementia is a progressively degenerative disease that is frequently encountered in general family practice. Diagnosis is best made following the collection of a comprehensive history and a thorough clinical examination that includes both physical and neurological investigation. The assessment recommendations outlined in this article would be of great assistance to family practitioners in gathering a large amount of valuable information regarding a patient's neurocognitive functioning. Multidisciplinary input from specialist neurology and mental-health professionals should also be sought to aid accurate diagnosis and the complete management of the illness. Finally, it is best borne in mind that the management of dementia should focus on both the identified patient and the caregiver.

\section{References}

1. Marsel Mesulam M. Principles of cognitive and behavioral neurology. New York: Oxford University Press; 2000.

2. Clark DG, Cummings JL. The diagnosis and management of dementia. Middle East J of Fam Med. 2004;5.

3. Dementia SA. Executive director's report on the activities of Dementia SA. 2009 June.

4. Säll L, Salamon E, Allgulander C, Owe-Larsson B. Psychiatric symptoms and disorders in HIV infected mine workers in South Africa: a retrospective descriptive study of acute first admissions. Afr J of Psychiatry. 2009;12:206-212.

5. World Health Organization. Joint United Nations programme on HIV/AIDS. Sub-Saharan Africa: latest epidemiological trends. 2009.

6. Petersen RC. Mild cognitive impairment clinical trials. Nature Rev Drug Discovery. 2003;2:646-653.

7. Diagnostic and statistical manual of mental disorders. 4th ed. Arlington: American Psychiatric Association; 2000.

8. Greeff OBW. Alzheimer's disease in family practice. SA Fam Pract. 2009;51(5):364-367.

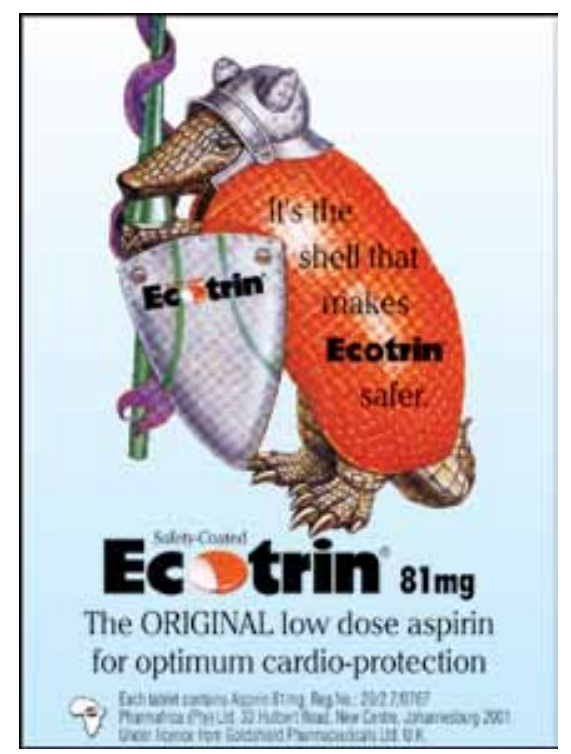

